\title{
Hábitos alimentarios, estado nutricional y estilos de vida en adultos mayores activos de la ciudad de Chillán, Chile
}

\author{
Eating habits, nutritional status \\ and lifestyle among active seniors \\ in the city of Chillan, Chile
}

\begin{abstract}
Aging is a public health issue that is becoming more relevant because eating habits and nutritional status are crucial in the lifestyle of seniors. The purpose of this work was to determine the eating habits, nutritional status, physical activity and lifestyle of active seniors belonging to three organized community groups in Chillán, Chile. This was a quantitative cross-sectional study that included 183 seniors. Eating habits and physical activity data were obtained using the Food-based Dietary Guidelines (Guías Alimentarias Basadas en los Alimentos, GABA). Evaluation of nutritional status was performed by anthropometry and BMI; lifestyle was evaluated with the FANTASTICO questionnaire. Data were analyzed by univariate and bivariate descriptive statistics and the Chi-square test using STATA 12.0 software at $\alpha=0.05$. A total of $65 \%$ and $32 \%$ of seniors had 3 and 4 daily mealtimes, respectively. On average, they consumed 5 portions of fruit and vegetables (60\%), 1 portion of dairy products $(87 \%)$, legumes (95\%), and fish (90\%). Daily water consumption was 6 to 8 glasses for $46 \%$ of seniors and $80 \%$ added salt to their meals and $42 \%$ did not consume saturated fats. All seniors (100\%) had some type of physical activity and 96\% classified their lifestyle as good or very good. Their nutritional status was mostly normal (52\%) and related to the intake of dairy products $(p=0,027)$. The active participation of seniors in organized community groups is a health-protective factor that promotes healthy lifestyles related to eating, physical activity, and an appropriate nutritional status, all of which stimulate healthy or successful aging.

Key words: Elderly; Eating habits; nutritional status; physical activity; lifestyle; active participation.
\end{abstract}

\section{INTRODUCCIÓN}

A nivel mundial actualmente, 600 millones de personas son adultos mayores $(A M)$ y se espera que esta cifra llegue a casi 2 mil millones para el año 2050 (1). En Chile, el 14,9\% de la población pertenece a este grupo, proyectándose para el año 2020 un 17.3\% de aumento con 1.788 .398 mujeres y 1.483.592 hombres (INE) (2). Actualmente, el envejecimiento se caracteriza por un retiro temprano del mercado laboral (60 años en la mujer y 65 en el hombre), seguido de un largo período de buena salud posterior al retiro. Al mismo tiempo,
Verónica Barrón

Alejandra Rodríguez

Pamela Chavarría

Departamento de Nutrición y Salud Pública, Facultad de Ciencias de la Salud y de los Alimentos, Universidad del Bío-Bío, Chillán, Chile.

Dirigir la correspondencia a: Alejandra Rodríguez Fernández Departamento de Nutrición y Salud Pública. Universidad del Bío-Bío, Sede Chillán. Avenida Andrés Bello S/N Chillán,

Teléfono/Fax: 56-42-2 463294 Email: alrodriguez@ubiobio.cl

Este trabajo fue recibido el 25 de Abril de 2016 y aceptado para ser publicado el 29 de Diciembre de 2016.

estas personas son más similares al resto de la población en términos de expectativas y motivaciones (3)

Es destacable que los AM en Chile sea el grupo de edad con mayor nivel de participación social, lo que se refleja en el aumento explosivo del número de organizaciones en los últimos años (4). La participación social es un elemento protector de la salud en los adultos mayores, al moderar los efectos negativos del estrés y contribuir así al bienestar y satisfacción con la vida (5). La mejoría de la calidad de vida de este grupo se debe en gran medida a los avances científicos como la 
medicina, la alimentación, la educación física, el deporte y la recreación. Actividades que han despertado gran interés del gobierno, lo que se ha concretado con la creación de planes y programas, apuntando a incentivar al grupo de personas adultas y adultos mayores a ser parte de diferentes tipos de actividades $(6,7)$

En los AM, los hábitos alimentarios son más heterogéneos que los del resto de la población y factores como el estado físico, medios materiales de que disponen, elementos psicológicos y sociales, pueden influir en ellos (8). Se ha demostrado como estos hábitos alimentarios inadecuados se convierten en un factor de riesgo importante de morbilidad y mortalidad contribuyendo a una mayor predisposición a infecciones y enfermedades crónicas y nutricionales asociadas con el envejecimiento, además de disminuir la calidad de vida de este colectivo humano, comprobando que son componentes fundamentales para la conservación de la autonomía funcional en el desarrollo de un envejecimiento exitoso (9). En este sentido, al evaluar la contribución de la buena nutrición a la prolongación de la vida, Centers for Disease Control and Prevention (CDC), señala que la longevidad depende en $19 \%$ de la genética, en un 10\% del acceso a atención médica, 20\% de factores ambientales como la contaminación y $51 \%$ de los factores de estilos de vida (10).

La Encuesta Nacional de Salud 2010 mostró un importante incremento de la obesidad y de la obesidad mórbida en $A M$ respecto a la Encuesta de 2003, donde, 2 de cada 3 adultos en Chile tienen exceso de peso con una mayor prevalencia de obesidad en mujeres y en personas con menor escolaridad. Específicamente, el grupo de 65 años presenta un $31 \%$ de obesidad y cerca del $90 \%$ es sedentaria (11). Estos indicadores son muy semejantes a los de América Latina con cifras de obesidad de 35\% en Montevideo, 20\% en Sao Paulo y en $17,7 \%$ en Argentina (12). Diversos estudios señalan la importancia del estado nutricional de la población en general, y de los ancianos en particular, como un indicador válido para predecir la longevidad y la calidad de vida en esta etapa del ciclo vital $(13,14)$.

La escasa información acerca de los hábitos alimentarios y estilos de vida en la escala nacional de este grupo de población y principalmente en la escala local, ponen de manifiesto la necesidad de contar con datos actualizados. Por ello, el objetivo de este trabajo es determinar los hábitos alimentarios, el estado nutricional y el estilo de vida de personas mayores activas pertenecientes a diferentes grupos organizados de la comunidad de Chillán.

\section{MATERIALES Y MÉTODOS Diseño}

Estudio cuantitativo, observacional, de corte transversal sobre los hábitos alimentarios (HA), estado nutricional (EN) y estilos de vida (EV) de adultos mayores. Se trabajó con el universo correspondiente a 183 AM activos de la ciudad de Chillán, los cuales asistían al menos 3 veces por semana a 3 centros de actividades recreativas y/o cognitivas, donde participaban en talleres de estimulación de la memoria, gimnasia aeróbica, baile entretenido y coro. En este proceso investigativo, se realizó el contacto formal con cada una de las instituciones en forma oral y escrita, según consideraciones del Comité de Ética de la Universidad del Bío-Bío. Cada uno de los participantes del estudio firmó un consentimiento informado.

Hábitos alimentarios y actividad física: se obtuvieron mediante un cuestionario basado en las Guías Alimentarias Basadas en los Alimentos (GABAs) del Ministerio de Salud
(2013) (13), incluyendo además datos de identificación sociodemográfica como la edad, género, estado civil y escolaridad.

Evaluación del estado nutricional: las mediciones antropométricas se realizaron con los criterios de Lohman y cols (15). Para la obtención del peso corporal se utilizó una balanza marca SECA® modelo 813, con capacidad de $200 \mathrm{~kg}$ y una precisión de $0,01 \mathrm{~kg}$, previamente calibrada. Para la talla se utilizó un tallímetro portátil marca SECA® modelo 213, con una precisión de $1 \mathrm{~mm}$ y un rango de medición de 20 a $205 \mathrm{~cm}$.

Para clasificar el estado nutricional se usó el Índice de Masa Corporal (IMC) para AM según referencia del MINSAL, aplicando los criterios: $I M C<$ a 23 enflaquecido, IMC entre 23,1-27,9 normal, IMC 28,0-31,9 sobrepeso y IMC >32 obesidad (16).

Estilo de vida

Se evaluó con el cuestionario FANTASTICO, instrumento genérico, diseñado en el Departamento de Medicina Familiar de la Universidad McMaster de Canadá, para ayudar a los profesionales de la salud a medir los estilos de vida. Ha sido validado en Colombia en 2001 (17) y adaptado en Chile, con autorización de la Universidad de Ontario Canadá, mediante el convenio de Promoción de Salud Guía para Universidades Saludables (18).

\section{Análisis de la información}

Se realizó utilizando estadística descriptiva uni y bivariada. De las variables cualitativas se obtuvo frecuencia absoluta y porcentajes representadas en tablas de distribución de frecuencias. Para las variables cuantitativas se usaron medidas de tendencia central y dispersión. La relación de variables se realizó con la prueba estadística Chi-cuadrado y Test exacto de Fisher, en el caso de frecuencias esperadas menores a 5. Se usó el programa STATA 12.0 utilizando un nivel de significancia $\alpha=0,05$.

\section{RESULTADOS}

Los 183 adultos mayores estudiados se caracterizaron por ser principalmente mujeres con un $88,5 \%$, no tener pareja (55\%) y presentar un nivel de escolaridad medio y superior del $87 \%$ (tabla 1). La edad y características antropométricas entre hombres y mujeres no fueron estadísticamente diferentes ( $p>$ $0,05)$, a excepción de la talla $(p=0,001)$. El promedio de edad fue de 71,4 $\pm 6,2$ años. El peso, talla e IMC de las mujeres fue de $66,9 \pm 10,1 \mathrm{~kg}, 1,54 \pm 0,06 \mathrm{~m}$ y $28,2 \pm 4,0 \mathrm{~kg} / \mathrm{m}^{2}$. Para los hombres estos valores fueron $69 \pm 9,7 \mathrm{~kg}, 1,6 \pm 0,06 \mathrm{~m}$ y $26,7 \pm 2,8$ $\mathrm{kg} / \mathrm{m}^{2}$. El estado nutricional muestra que tanto en hombres como en mujeres predomina el normo peso (57,3 y 53,7\%), seguido de sobrepeso $(38,1$ y $30,2 \%)$ y obesidad $(4,8$ y 16,1), respectivamente. Dichos resultados no son significativamente diferentes $(p=0,365)$ (tabla 2$)$.

Respecto a los hábitos alimentarios se encontró que el $66 \%$ realizaba 3 tiempos de comida al día y un 34\% consumía las 4 . El 68\% refirió consumir 1 a 2 porciones de lácteos, el $19 \%$ más de 2 y el $13 \%$ no consume. De quienes sí lo hacían, el $62 \%$ era bajo en grasa, el $27 \%$ medio y $11 \%$ alto en grasa. El consumo adecuado de legumbres de 2 o más veces por semana, alcanzó el $45 \%$, el $49 \%$ consume 1 vez y sólo el $6 \%$ no consume. El 15\% consume 2 o más porciones de pescado por semana, el $75 \%$ consume 1 porción y el $10 \%$ no consume. La preparación más frecuente fue cocida o al horno, con un $97 \%$, sólo el 3\% lo consume frito. La distribución del consumo de frutas fue 56\% 1 a 2 porciones y 44\%, 2 o más; el consumo de verduras revela que aunque sólo el $17 \%$ cumple 
la recomendación de 2 a 3 porciones diarias, el $83 \%$ restante consume 1 a 2 . Respecto a la ingesta de agua, el $46 \%$ consumía 6 a 8 vasos al día, el $47 \%$ de 3 a 5 . El consumo extra de sal, refleja que el $80 \%$ agrega sal adicional, el $14 \%$ no lo hace y el $6 \%$ ocasionalmente. Al consultar por el consumo de alimentos ricos en grasas saturadas (mantequilla, embutidos, frituras), el 43\% dice no consumir (tabla 3 ).

En relación a la actividad física el 93\% refiere hacer algún tipo de actividad, como yoga, gimnasia, baile entretenido y caminar al menos 30 minutos al día. Al evaluar el estilo de vida, el $96 \%$ clasificó con estilo de vida muy bueno y bueno.

Al relacionar el estado nutricional con los hábitos alimentarios, la actividad física y el estilo de vida, sólo se encontraron diferencias estadísticamente significativas con el consumo de lácteos (tabla 3). Los que cumplen la recomendación de más de 2 porciones de lácteos, alcanzaron un $77 \%$ de normalidad por sobre el $47,8 \%$ que no consume y el $48,8 \%$ que consume de 1 a 2 porciones. Aunque no hay diferencias significativas entre los tiempos de comida, se observa en quienes consumen 4 comidas, un $62 \%$ de normalidad, por sobre el $50 \%$ de los que consumen 3. Los $A M$ que no consumen legumbres en forma adecuada, presentan menos sobrepeso $(29,7 \%)$ que los que consumen 1 vez por semana $(32,2 \%)$. Lo mismo ocurre con las frutas y verduras. La mayor proporción de obesidad se aprecia en los que consumen pescado 1 vez por semana (16,1\%). El mayor consumo de agua muestra mayor prevalencia de estado nutricional normal, $61 \%$ en quienes consumen de 6 a 8 vasos, contra 50,0\% en los que consumen de 3 a 5 vasos. El 53,5\% de los AM que realiza algún tipo de actividad física tienen EN normal, y quienes no realizan presentan $23,1 \%$ de obesidad. Las personas con estilo de vida bueno y muy bueno presentan los porcentajes más altos de estado nutricional normal.

\section{DISCUSIÓN}

La calidad de vida y longevidad de las personas mayores depende en gran parte de los hábitos de alimentación y diferentes factores de tipo psico-social, que determinan la seguridad alimentaria y nutricional, como la soledad, falta de recursos, baja disponibilidad de alimentos, anorexia y enfermedades crónicas entre otras, las cuales determinan el consumo

TABLA 1

Características socio-demográficas de los adultos mayores estudiados.

\begin{tabular}{|c|c|c|c|c|}
\hline \multirow[t]{2}{*}{ Variables } & \multirow[t]{2}{*}{$\mathrm{N}$} & \multirow[t]{2}{*}{$\%$} & \multicolumn{2}{|c|}{ IC 95\% } \\
\hline & & & Linf & Lsup \\
\hline \multicolumn{5}{|l|}{ Sexo } \\
\hline Masculino & 21 & 11,5 & 6,8 & 16,1 \\
\hline \multicolumn{5}{|c|}{ Condición de pareja } \\
\hline \multicolumn{5}{|l|}{ Escolaridad } \\
\hline Básica & 24 & 13,0 & 8,2 & 18,0 \\
\hline Media & 86 & 47,0 & 39,7 & 54,3 \\
\hline Superior & 72 & 40,0 & 32,7 & 47,1 \\
\hline
\end{tabular}

\section{TABLA 2}

Características antropométricas y estado nutricional de adultos mayores según género.

\begin{tabular}{lccc}
\hline Antropometría & Femenino & Masculino & $\mathrm{p}$ \\
& $\bar{\chi}$ (D.E) & $\bar{\chi}(\mathrm{D} . \mathrm{E})$ & $0,806^{1}$ \\
Edad (años) & $71,3 \pm 6,4$ & $71,7 \pm 5,1$ & $0,365^{1}$ \\
Peso (Kg) & $66,9 \pm 10,1$ & $69,0 \pm 9,7$ & $0,0001^{1}$ \\
Talla (m) & $1,54 \pm 0,06$ & $1,60 \pm 0,06$ & $0,099^{1}$ \\
IMC & $28,2 \pm 4,1$ & $26,7 \pm 2,8$ & $0,365^{2}$ \\
Estado nutricional & $\%(\mathrm{~N})$ & $\%(\mathrm{~N})$ & \\
Normal & $53,7(87)$ & $38,1(12)$ & $4,8(1)$ \\
Sobrepeso & $30,2(49)$ & $100(21)$ & \\
Obeso & $16,1(26)$ & \\
Total & $100(162)$ & & \\
\hline${ }^{1}$ T-student & & & \\
${ }^{2}$ Chi cuadrado & &
\end{tabular}


de alimentos y el estado nutricional $(19,20)$.

Los resultados reflejan que la mayoría de los $A M$ realiza 3 tiempos de comida (desayuno, almuerzo y once-comida o comida) y aunque el 34\% incluye la cena, este porcentaje es insuficiente considerando que incluirla podría minimizar el riesgo de déficit en el consumo de calorías y nutrientes asociados a los procesos de fragilización (19).

El $61 \%$ de adecuación del consumo de 5 porciones de frutas y verduras al día, fue superior a los resultados de la Encuesta Nacional de Consumo Alimentario, ENCA (21) del $51 \%$ en este grupo etario y a la Encuesta Nacional de Salud 2010 que revela un $17 \%$ de consumo adecuado. Las frutas y verduras son determinantes en el aporte de fibra, a la que se le reconocen beneficios fisiológicos y metabólicos. Estudios han demostrado que un aumento en el consumo de fibra soluble de 3 a 10 gr/día disminuye significativamente el colesterol total sanguíneo y las concentraciones de LDL, sin cambiar significativamente las concentraciones de triglicéridos y $\mathrm{HDL}$
(22) y por otra parte, se ha comprobado que previenen la hipertensión arterial, dislipidemia y diabetes, enfermedades de alta prevalencia en este grupo de edad (23).

El consumo de lácteos indicó el menor porcentaje de adecuación de los alimentos evaluados respecto a la recomendación de 2 o más porciones diarias, con un 19\%, lo que se presenta de igual forma en la ENCA en este grupo etario. Sin embargo, a pesar de que existe un déficit respecto a la recomendación, es importante destacar que el $68 \%$ consume una a dos porciones al día y la mayoría es baja en grasa. La baja ingesta de calcio genera pérdida mineral ósea y un mayor riesgo de fracturas, ya que el calcio y la vitamina $D$ protegen contra la osteoporosis y reducen la tasa de pérdida ósea en los últimos años de vida (24). El consumo de leguminosas una a dos veces por semana alcanzó a un 94\%, muy superior al $29 \%$ de consumo que señala la ENCA en el grupo de 65 años y más. Estas son conocidas como buena fuente de proteína vegetal y carbohidratos, buen aporte de fibra, vitaminas del

\section{TABLA 3}

Estado nutricional de los adultos mayores según hábitos alimentarios.

\begin{tabular}{|c|c|c|c|c|c|}
\hline \multirow[b]{2}{*}{$\begin{array}{c}\text { Hábitos } \\
\text { Alimentarios }\end{array}$} & \multicolumn{4}{|c|}{ Estado nutricional } & \multirow[b]{2}{*}{$\mathrm{p}$} \\
\hline & $\begin{array}{l}\text { Normal } \\
\%(\mathrm{~N})\end{array}$ & $\begin{array}{c}\text { Sobrepeso } \\
\%(N)\end{array}$ & $\begin{array}{l}\text { Obeso } \\
\%(\mathrm{~N})\end{array}$ & $\begin{array}{l}\text { Total } \\
\%(\mathrm{~N})\end{array}$ & \\
\hline $\begin{array}{l}\text { Tiempo de comid } \\
3 \\
4\end{array}$ & $\begin{array}{l}50,0(60) \\
61,9(39)\end{array}$ & $\begin{array}{l}32,5(39) \\
28,6(18)\end{array}$ & $\begin{array}{c}17,5(21) \\
9,5(6)\end{array}$ & $\begin{array}{c}66,0(120) \\
34,0(63)\end{array}$ & 0,216 \\
\hline $\begin{array}{l}\text { Lácteos (porción/ } \\
1 \text { a } 2 \\
>3 \\
\text { No consume }\end{array}$ & $\begin{array}{l}48,8(61) \\
77,2(27) \\
47,8(11)\end{array}$ & $\begin{array}{c}36,8(46) \\
11,4(4) \\
30,4(7)\end{array}$ & $\begin{array}{l}14,4(18) \\
11,4(4) \\
21,8(5)\end{array}$ & $\begin{array}{c}68,0(125) \\
19,0(35) \\
13,0(23)\end{array}$ & $0,027^{*}$ \\
\hline $\begin{array}{l}\text { Legumbres (vez/s } \\
1 \mathrm{v} / \mathrm{s} \\
2 \text { o más v/s } \\
\text { No consume }\end{array}$ & $\begin{array}{l}54,4(49) \\
51,4(38) \\
80,0(8)\end{array}$ & $\begin{array}{c}32,2(29) \\
29,7(22) \\
10,0(1)\end{array}$ & $\begin{array}{c}13,4(12) \\
18,9(14) \\
10,0(1)\end{array}$ & $\begin{array}{c}49,0(90) \\
45,0(83) \\
6,0(10)\end{array}$ & 0,302 \\
\hline $\begin{array}{l}\text { Frutas (porción/di } \\
1 \text { a } 2 \\
>3\end{array}$ & $\begin{array}{l}55,9(57) \\
51,9(42)\end{array}$ & $\begin{array}{l}32,4(33) \\
29,6(24)\end{array}$ & $\begin{array}{l}11,7(12) \\
18,5(15)\end{array}$ & $\begin{array}{c}56,0(102) \\
44,0(81)\end{array}$ & 0,441 \\
\hline $\begin{array}{l}\text { Verduras (porción } \\
1 \text { a } 2 \\
>2\end{array}$ & $\begin{array}{l}53,3(81) \\
59,1(18)\end{array}$ & $\begin{array}{c}32,2(49) \\
25,8(8)\end{array}$ & $\begin{array}{c}14,5(22) \\
16,1(5)\end{array}$ & $\begin{array}{c}83,0(152) \\
17,0(31)\end{array}$ & 0,768 \\
\hline $\begin{array}{l}\text { Pescado } \\
1 \mathrm{v} / \mathrm{s} \\
2 \text { o más } \\
\text { No consume }\end{array}$ & $\begin{array}{l}53,3(73) \\
50,0(14) \\
66,7(12)\end{array}$ & $\begin{array}{c}30,6(42) \\
35,7(10) \\
27,8(5)\end{array}$ & $\begin{array}{c}16,1(22) \\
14,3(4) \\
5,5(1)\end{array}$ & $\begin{array}{c}75,0(137) \\
15,0(28) \\
10,0(18)\end{array}$ & 0,720 \\
\hline $\begin{array}{l}\text { Agua (vasos/día) } \\
1 \text { a } 3 \\
3 \text { a } 5 \\
6 \text { a } 8\end{array}$ & $\begin{array}{c}38,5(5) \\
50,0(55) \\
61,0(51)\end{array}$ & $\begin{array}{l}53,9(7) \\
34,0(31) \\
25,0(21)\end{array}$ & $\begin{array}{c}7,6(1) \\
16,0(19) \\
14,0(12)\end{array}$ & $\begin{array}{l}7,0(13) \\
47,0(86) \\
46,0(84)\end{array}$ & 0,242 \\
\hline $\begin{array}{l}\text { Sal extra } \\
\text { No } \\
\text { Ocasionalmente } \\
\text { Si }\end{array}$ & $\begin{array}{c}50,0(12) \\
66,7(8) \\
53,7(79)\end{array}$ & $\begin{array}{l}29,2(7) \\
16,7(2) \\
32,7(48)\end{array}$ & $\begin{array}{c}20,8(5) \\
16,6(2) \\
13,0(24)\end{array}$ & $\begin{array}{c}13,6(20) \\
7,0(12) \\
80,0(147)\end{array}$ & 0,711 \\
\hline Total & $54,1(99)$ & $31,1(57)$ & $14,8(27)$ & 183 & \\
\hline
\end{tabular}


complejo B, especialmente B3 y ácido fólico y minerales como hierro, calcio y fósforo, destacándose su excelente relación sodio/potasio superior incluso en la relación existente en nueces y paltas (25).

El consumo de pescado alcanzó un $90 \%$ a lo menos una vez a la semana y solo el 3\% lo consume frito, mientras que la ENCA muestra un 13\% de cumplimiento de recomendación para el grupo del adulto mayor. Este alimento es considerado el más sano de las carnes blancas, bajo en grasas y calorías, excelente fuente de vitaminas del complejo $B$, satisfaciendo el $100 \%$ de necesidades diarias de vitamina D. Su consumo disminuye el nivel de colesterol sanguíneo y de triglicéridos, reduciendo así el riesgo de enfermedades cardiovasculares, aumentando el desarrollo cognitivo en todas las etapas de la vida (26)

El $80 \%$ del grupo estudiado agrega sal adicional a las comidas. El consumo excesivo de sal es un problema de toda la población y los datos actuales indican que esto es particularmente importante en los adultos mayores. Cerca del 55\% en las mujeres y el $49 \%$ en hombres entre 55 y 64 años son hipertensos. Estos valores aumentan al $74 \%$ y el $64 \%$ entre los 65 y 74 años, respectivamente (26). La reducción de sodio en la dieta de los adultos mayores disminuye la presión arterial y se ha asociado con una menor incidencia de accidentes cerebrovasculares y de enfermedades cardíacas (27). El consumo de 6 a 8 vasos de agua recomendados la cumple el $46 \%$ del grupo, lo que es importante, considerando que las personas mayores están en constante riesgo de deshidratación, debido a la disminución del agua corporal total y una clara disminución de la percepción de la sed y capacidad para concentrar la orina (26).

El estado nutricional normal predominante entre hombres y mujeres, sería el reflejo de la buena adecuación de los hábitos alimentarios en este grupo de estudio, a diferencia de la alta prevalencia de sobrepeso y obesidad de adultos mayores reportados en diferentes investigaciones (28, 29, $30,31)$. A lo anterior, se suma el alto porcentaje de $A M$ que realiza algún tipo de actividad física, que es el factor que más se asocia con una buena capacidad funcional hasta el final de la vida. Los hombres y mujeres físicamente activos a partir de los 50 años, viven uno a tres años más sin trastornos cardiovasculares en comparación con adultos inactivos, promueve un mejor balance energético con mantenimiento del peso y una composición corporal más favorable (32). Varios estudios con personas mayores han indicado que el ejercicio físico está asociado a un menor declive cognitivo (33), o al menos que la participación en determinados programas de ejercicio físico ha repercutido, positiva y moderadamente en el rendimiento en evaluaciones de la función cognitiva $(34,35)$.

El haber encontrado un $96 \%$ de clasificación con estilo de vida bueno y muy bueno, estaría evidenciando el buen nivel de estudios del grupo, que los motivaría a la participación social activa en grupos organizados de la comunidad, lo que sería un promotor de buenos hábitos alimentarios, de actividad física y estado nutricional normal, reflejándose en un envejecimiento exitoso y contribuyendo a una mejor calidad de vida de este grupo etario.

Aunque los resultados de esta investigación son sólo atingentes al grupo de estudio, se esperaría que $A M$ con las mismas características de nivel educacional y participación activa presentaran un patrón similar respecto a las variables estudiadas. En este sentido, la mayor posibilidad de las personas mayores por mantener estilos de vida saludables, estaría principalmente asociada a la educación. Es por esto que se recomienda intervenir en los grupos menos favorecidos edu- cacionalmente, respecto de hábitos saludables y participación activa como protectores de la salud.

\section{RESUMEN}

El envejecimiento es un tema de salud pública cada vez más relevante, siendo los hábitos alimentarios y el estado nutricional fundamentales en el estilo de vida de los adultos mayores (AM). El objetivo de este trabajo fue determinar hábitos alimentarios, estado nutricional, actividad física y estilo de vida de $A M$ activos pertenecientes a 3 grupos organizados de la comunidad de Chillán. Estudio cuantitativo de corte transversal con una muestra de 183 AM. Los hábitos alimentarios y la actividad física se evaluaron mediante un instrumento basado en las Guías Alimentarias actuales (GABAs). La evaluación del estado nutricional mediante antropometría e IMC y el estilo de vida con el cuestionario FANTASTICO. Los datos fueron analizados con estadística descriptiva univariada, bivariada y con la prueba de chi-cuadrado en el programa STATA 12.0 con $\alpha=0,05$. El $65 \%$ y $32 \%$ de los $A M$ realizaban 3 y 4 tiempos de comida respectivamente. En promedio, consumían 5 porciones de frutas y verduras (60\%) y una porción de lácteos el (87\%), legumbres (95\%), pescados (90\%). El 46\% consumía 6 a 8 vasos de agua al día. El $80 \%$ usaba sal extra y el $42 \%$ no consumía grasas saturadas. El 100\% realizaba algún tipo de actividad física y el $96 \%$ clasificó en estilo de vida bueno y muy bueno. El estado nutricional predominante fue normal (52\%), el cual se relacionó con el consumo de lácteos $(p=0,027)$. La participación social de AM en grupos organizados de la comunidad, es un elemento protector de la salud que promueve estilos de vida saludables relacionados a la alimentación, actividad física y adecuado estado nutricional, favoreciendo un envejecimiento saludable o exitoso.

Palabras clave: Hábitos alimentarios; estado nutricional; actividad física; estilo de vida; participación activa.

Agradecimientos: A la Universidad del Bío-Bío, Chile (Proyecto DIUBB 143920 2/R), por su apoyo en la ejecución de este proyecto. Al Centro de Actividades Prácticas (CAP) Carrera de Fonoaudiología, Universidad del Bío-Bío, Instituto de Previsión Social (IPS) y Agrupación Adultos Mayores John Kennedy, por su colaboración y participación.

\section{REFERENCIAS}

1. World Health Organization. Ageing and the Life-course. International Day of Older Persons. Retrieved August 2, 2014 from http://www.who.int/ageing/es/

2. National Institute of Statistics (INE), Government of Chile, Chile.

3. Walker A. Understanding Quality of life in Old Age. Maidenhead: Open University Press 2005.

4. National Service for Older Adults (SENAMA), Government of Chile. Active Aging. http://www.senama.cl/EnvejecimientoAct.html

5. Aguirre C, Bouffard, L. Successful Aging: Theories, Research Studies, and Clinical Applications. Rev Asoc Colombiana Gerontología y Geriatría. 2008; 22:1146-62.

6. Osorio P, Torrejón MJ, Anigstein MS. Quality of Life of Older Adults in Chile. Rev Mad, Universidad de Chile. 2011; 24:61-75.

7. Fernández $N$, Clúa, A Báez, $R$ \& Ramírez M. Lifestyles, Subjective Wellness, and Health of the Elderly. Rev Cubana Med Gral. 2000; 16:6-12.

8. Ávila J., Garant M., Savarro S. Relationship between Factors That Determine the Symptoms of Depression and Eating 
Habits of Older Adults in Mexico. Rev Panamericana de Salud Pública. 2006; 19(5):321-30

9. Alvarez J, Gonzalo I, Rodríguez JM. Ageing and Nutrition. Nutr Hosp. 2011;:4(3):3-14

10. Krinke B. Nutrition and Older Adults, in: Nutrition in the Different Stages of Life. Chapter 18. Mexico City. MacGraw Hill.pp 456-482, 2010.

11. Ministry of Health. National Health Survey. 2010.

12. Acosta L, Carrizo D, Peláez E, Roque B. Life Conditions, Nutritional Status, and Health Status of Older Adults. Cordoba, Argentina. Retrieved from http://www.scielo. br/pdf/rbgg/v18n1/1809-9823-rbgg-18-01-00107.pdf

13. Ministry of Health. Department of Nutrition and Food. Educational Guide. Food Guides for the Population. 2013.

14. Muñoz, L. V. A., Ibáñez, M. E. Quality of life and healthy food habits and education of elderly persons. Rev. Humanidades. 2015; 25:145-168.

15. Lohman T.G, Roche AF, Martorell R. Anthropometric Standardization Reference Manual. USA, Human Kinetics Books. pp 184, 1988.

16. Ministry of Health, Chile, 2002. Nutrition in the Life Cycle. Cornejo V. Cruchet S. Ed. Mediterráneo. pp 124, 2014.

17. Robinson $R$, Agredo $R$. Reliability and Validity of the "FANTÁSTICO" Instrument to Measure the Lifestyle of Columbian Adults. Physical Exercise and Sports Research Group. Physiotherapy Program, Universidad Manuela Beltrán. 2012.

18. Lange I, Vio F. Guide for Healthy Universities and Other Postsecondary Institutions. 2006 Retrieved November 11, 2016 from http://www7.uc.cl/ucsaludable/img/guiaUSal. pdf.

19. Restrepo S, Morales R.M, Ramírez M, López V, Varela L. Nutritional Habits in Senior Adults and Its Relationship with Protective or Deteriorating Effects in Health. Rev Chil Nutr. 2006; 33:(3):500-10.

20. Barrón V, Vera S, Ojeda G, Mardones H. Comparison of Lyfestyles for the Economically Active Population Older than 65 Years in the Cities of Chillán and Valparaíso, Chile. Theoría. 2006; 15:33-44

21. National Food Consumption Survey Encuesta Nacional de Consumo Alimentario ENCA. http://web.minsal.cl/sites/ default/files/ENCA-INFORME_FINAL.pdf

22. Davy B, Melby $C$. The effect of fiber-rich carbohydrates on features of Syndrome X. J Am Diet Assoc. 2003; 103:86-96.

23. Mediterranean Diet and Disease Prevention. Healthy Medi- terranean gastronomy digital magazine (periodic online publication, URL) http://wwwsabormediterráneo. como/ salud/dietemediterranea1.htm.

24. González-Solanellas M, Romagosa Pérez-Portabella A, Zabaleta-del-Olmo E, Grau-Carod M, Casellas-Montagut C, Lancho-Lancho S. et al. Prevalence Study of Eating Habits and Nutritional Status of Adult Population Treated in Primary Care. Nutr Hosp. 2011; 26(2):337-44.

25. Salazar A M. Nutrition in the Older Adult, in: Nutrition in the Life Cycle. Cornejo V. Cruchet S. Ed. Mediterráneo. pp 137-8, 2014.

26. Serrano M, Cervera P, López C, Rivera J.M, Sastre A. Food Guide for Older Persons. Spanish Centre for Reprographic Rights, CEDRO, 2010. www.cedro.org.

27. World Health Organization (WHO) http://www.who.int/ elena/titles/sodium cvd_adults/es/

28. Sánchez S, García C, Duque M, Juarez T, Cortés A, Reyes S. Anthropometric measures and nutritional status in a healthy elderly population. BMC Public Health. 2007; 7:2

29. Sánchez F, De la Cruz F, Cereceda M, Espinoza S. Association of dietary habits and nutritional status with socioeconomic status of older adults attending a Municipal Program. An Fac Med. 2014; 75(2):107-11

30. Zaragoza $A$, Ferrer $R$, Cabañero $M^{a} J$, Hurtado JA, Laguna A. Adherence to the Mediterranean Diet and Its Relation to Nutritional Status In Older People Nutr Hosp 2015;31(4):1667-1674 Retrieved December 19, 2016 from <http://Www.redalyc.org/ articulo. oa?id=309238513029> ISSN 0212-1611

31. Martinez Tomé MJ, Rodriguez A, Jimenez AM, Mariscal M, Murcia MA, García-Diz L. Food habits and nutritional status of elderly people living in a Spanish Mediterranean city. Nutr Hosp. 2011; 26:1175-82

32. Franco, O. H., de Laet, A. Peeters, J. Mackenbach y W.Nusselder. Effects of physical activity on life expectancy with cardiovascular disease. Arch Intern Med. 2001; 165:2355-60

33. Leatle $M$, Vander $B$, Pandav $R$, Dodge $H$, Ganguly $M$. Exercise level and cognitive decline: themoviesproject. Alzheimer Dis Assoc Disord. 2004; 18:57-64

34. Pandav R, Dodge H, Ganguly M. Exercise level and cognitive decline: the movies project. Alzheimer Dis Assoc Disord. 2004; 18:57-64

35. Colombe S, Kramer A. Fitness effects on the cognitive function of older adults: a meta-analytic study. Psychol Sci. 2003; 14:125-130. 